\title{
Risk factors for functional deterioration in a cohort with late effects of poliomyelitis: A ten-year follow-up study
}

\author{
Zeev Meiner ${ }^{\mathrm{a}, *}$, Anat Marmor ${ }^{\mathrm{a}}$, Murad Jalagel ${ }^{\mathrm{a}}$, Hagai Levine ${ }^{\mathrm{b}}$, \\ Shimon Shiri ${ }^{\mathrm{a}}$ and Isabella Schwartz ${ }^{\mathrm{a}}$ \\ ${ }^{a}$ Department of Physical Medicine and Rehabilitation, Hadassah Medical Center and Faculty of Medicine, \\ Hebrew University of Jerusalem, Jerusalem, Israel \\ ${ }^{\mathrm{b}}$ Braun School of Public Health, Hebrew University-Hadassah, Jerusalem, Israel
}

Received 27 July 2021

Accepted 6 August 2021

\begin{abstract}
.
BACKGROUND: More than 7000 patients developed poliomyelitis during the main epidemic in the fifties in Israel. In recent years, there is a further deterioration in their condition due to accelerated aging process and post-polio syndrome.

OBJECTIVE: To evaluate the risk factors for the progression of functional status in a cohort of patients with late effect of poliomyelitis over a period of ten years.

METHODS: A cross-sectional cohort study including 82 individuals with late effect of poliomyelitis evaluated over ten years. Mean age was $67 \pm 8.5$ years, $52.4 \%$ were men and $79.3 \%$ were Jewish. Functional status was evaluated by activities of daily living (ADL) questionnaire. Risk factors, including general comorbidities, history of poliomyelitis infection, use of assistive devices, employment, and physical activity statuses were evaluated using specific questionnaires.

RESULTS: Independence in ADL functions deteriorated significantly over ten years. Older age, ethnicity, use of a wheelchair, and use of orthotic devices in childhood were risk factors for deterioration in ADL function. No correlation was found between the presence of other comorbidities or poliomyelitis parameters and worsening of ADL functions.

CONCLUSIONS: Late effect of poliomyelitis was associated with deterioration in ADL functions probably due to the combined effect of the initial severity of the paralytic poliomyelitis symptoms and accelerated aging.
\end{abstract}

Keywords: Poliomyelitis, functional outcomes, ADL, wheelchair use

\section{Introduction}

Poliomyelitis was a substantial public health threat in the 20th century, before the development of the polio vaccine (Halstaed, 2011). Millions of people, most of them children, were struck by the disease and left with many disabilities that have affected

\footnotetext{
*Address for correspondence: Zeev Meiner, M.D., Department of Physical Medicine and Rehabilitation, Hadassah University Hospital, PO Box 24035, Jerusalem 91240, Israel. Tel.: +972 2 5844474; Fax:+972 2 5829113; E-mail: meiner@hadassah.org.il.
}

their mobility and quality of life. According to the WHO, it is estimated that there are 10-20 million of poliomyelitis patients worldwide (Gonzalez \& Olsson \& Borg, 2010). In Israel, more than 7,000 people have contracted poliomyelitis, most of them during the years of the widespread epidemics, 1949-1956, and usually in their childhood (Adler \& Eliakim \& Magora, 1955; Slater \& Handsher, 1989; Swartz, 2008).

These people, most of whom are now in their 60 s and 70 s, after having been clinically stable for 
many years, have started to suffer from a deterioration of their condition, which is harmful to their general health and functioning (Jacob \& Shapira, 2010; McNalley \& Yorkston \& Jensen \& Truitt \& Schomer, 2015). One of the possible causes of this decline is known as post-polio syndrome (PPS), which has a deleterious effect on the function and quality of life of patients (Ahstrom \& Karlsson, 2000; Dalakas \& Elder \& Hallett, 1986; Trojan \& Cashman, 2005) [8-10]. Several approaches have been used to diagnose PPS, most of which are based on muscle atrophy or dysfunction (Li Hi Shing et al., 2019). Another possible reason for this decline is an accelerated aging process in this population (Lo \& Robinson, 2018).

Several studies have shown that patients with late effect of poliomyelitis (LEoP) are more prone to develop cardiovascular disease (CVD), hypertension, diabetes, and chronic pain than the general population (Kang \& Lin, 2011; Nielsen \& Rostgaard \& Askgaard \& Skinhøj \& Aaby, 2004; Schwartz et al., 2018). It is still not known, however, whether this high prevalence of morbidity is associated with a higher rate of progression of clinical symptoms and functional deterioration. It is also not clear whether the history of the primary poliomyelitis infection, including the extent of disease involvement and the level of primary disability, are associated with a higher rate of disability progression.

In our previous work, ten years ago, we showed that higher disability in activities of daily living (ADL) and mobility were associated with higher severity of poliomyelitis sequelae (Schwartz et al., 2014). Since then, the poliomyelitis population grew older, and their clinical and functional level have declined. The aim of this study was to investigate the severity of the deterioration in the functional level of patients with LEoP, and to identify the risk factors associated with this progression, including demographic parameters, general comorbidities, and history of poliomyelitis characteristics.

\section{Materials and methods}

\subsection{Participants}

Participants in the study were 82 patients who attended the post-polio clinic in the Physical Medicine and Rehabilitation Department at Hadassah Mount Scopus Medical Center in Jerusalem, and participated in our previous study, ten years earlier (Schwartz et al., 2014). The inclusion criterion was a diagnosis of previous paralytic poliomyelitis, with confirmation by clinical examination and EMG. Cognitive ability to participate in the study was evaluated using the short portable mental status questionnaire (SPMSQ) (Pfeiffer, 1975). The exclusion criterion was evidence of other neurological diseases that could explain the neurological impairment. A message was sent to all 197 patients who participated in the previous study, in 2009, and those who answered were scheduled for a phone interview to complete the survey. The study was approved by the Institutional Review Board of Hadassah Medical Center. All participants provided consent by agreeing to complete the questionnaires.

\subsection{Survey instruments}

\subsubsection{Demographic, comorbidities and poliomyelitis history questionnaire}

Most of the information was based on the survey questionnaire used in our previous study, which included questions to collect demographic, medical, social, and functional information (Schwartz et al., 2018). The demographic data included current age, age at polio infection, gender, ethnic origin, and place of birth (Israel or abroad). Comorbidity data, including heart disease, high blood pressure, diabetes mellitus, lung diseases, thyroid disease, obesity, current and past smoking, and chronic back or neck pain, were obtained from a comorbidity questionnaire. The characteristics of the original poliomyelitis infection included age at polio infection, categorized into three subgroups based on motor neurodevelopment stages: below one year, between two and three years, and above three years; The distribution of the muscle weakness, whether it involved the upper or lower limbs or both; involvement of the respiratory system; orthopedic surgery in childhood; and use of orthotics in childhood. The characteristics of the severity of polio sequelae included the presence of scoliosis, use of orthotics in adulthood, use of wheelchair, being wheelchair-bound, and use of motorized wheelchair. A new questionnaire was sent to the participants, including updates on marital status, occupational status, and physical activities.

\subsubsection{Functional status questionnaire}

A self-report questionnaire was administered to identify difficulties in performing activities of daily living (ADL). The ADL questionnaire assesses the level of independence in bathing/showering, dressing, eating, and functional mobility, which includes 
mobility in bed, sit-to-stand transfers, and indoor and outdoor mobility. The patients' overall functioning was assigned a grade on a five-point scale: $1=$ complete independence, 2 = independent with difficulties, $3=$ independent with assistance, $4=$ requiring personal assistance, and $5=$ fully dependent. Participants were also asked about walking aids and wheelchair use.

\subsection{Statistical methods}

Descriptive statistics for continuous variables are presented as means and standard deviations (SD), and categorical variables as frequencies and percentages. We used a two-sided Wald test to compare responses provided in 2009 and 2020 for categorical data (there were no continuous comparisons required at this stage). We calculated average total ADL scores and scores for each element separately, for 2009 and 2020. We compared the averages using two-sided paired $t$-tests. We also calculated the average change in ADL between the years for different subgroups, and compared the changes using two-sided $t$-tests. We conducted a linear regression for the change in ADL between 2009 and 2020, adjusting for different demographic, functional, and medical parameters separately. A final regression included all parameters with a statistical significance of $p<0.3$. Statistical significance was set at $p<0.05$. All analyses were performed using R 3.5.0.

\section{Results}

\subsection{Demographics, comorbidities, and vascular risk factors}

The demographic data of the cohort of 82 patients with LEoP, followed up for ten years, is shown in Table 1. In 2020, the mean age of the cohort was 67 years, $52.4 \%$ were men, and $79.3 \%$ were Jews. A third were still employed, similarly to the 2009 data, and the same percentage of the cohort were still physically active.

The prevalence of comorbidities (hypertension, diabetes, heart and lung disease), of vascular risk factors (smoking and BMI), and of the characteristics of poliomyelitis found in 2009 are presented in Table 2. High percentage of our participants presented vascular risk factors such as obesity, smoking, and sedentary life style, and vascular comorbidities
Table 1

Demographic data of 82 participants

\begin{tabular}{lccc}
\hline Demographic & 2009 & 2020 & $P$ \\
\hline Age (years) (Mean \pm SD) & $57.2 \pm 8.8$ & $67 \pm 8.5$ & \\
Male & $52.4 \%$ & $52.4 \%$ & \\
Jews & $79.3 \%$ & $79.3 \%$ & \\
Married & $71 \%$ & $66 \%$ & 0.6147 \\
Education (years) (Mean \pm SD) & $13.5 \pm 4.3$ & & \\
Physical activity in previous week & $40.2 \%$ & $39 \%$ & 0.999 \\
Working in previous week & $43.9 \%$ & $30.5 \%$ & 0.1062 \\
\hline
\end{tabular}

Table 2

Comorbidities, vascular risk factors, and poliomyelitis characteristics of 82 participants in 2009

\begin{tabular}{|c|c|c|}
\hline \multirow{10}{*}{$\begin{array}{l}\text { Vascular risk } \\
\text { factors and } \\
\text { comorbidities }\end{array}$} & $\mathrm{BMI}(\mathrm{Mean} \pm \mathrm{SD})$ & $26.85 \pm 4.8$ \\
\hline & Obesity- BMI > 30 & $22 \%$ \\
\hline & Smoking & \\
\hline & Before 2009 & $39 \%$ \\
\hline & In 2009 & $21 \%$ \\
\hline & Absence of physical activity & $59.8 \%$ \\
\hline & Hypertension & $39 \%$ \\
\hline & Diabetes & $28 \%$ \\
\hline & Heart attack & $8.5 \%$ \\
\hline & Coronary heart disease & $30.5 \%$ \\
\hline \multirow{4}{*}{$\begin{array}{l}\text { Other } \\
\text { comorbidities }\end{array}$} & Chronic lung disease & $12.3 \%$ \\
\hline & Ulcer and intestinal diseases & $19.5 \%$ \\
\hline & Thyroid disease & $19.5 \%$ \\
\hline & Chronic pain & $63.4 \%$ \\
\hline \multirow{10}{*}{$\begin{array}{l}\text { Poliomyelitis } \\
\text { characteristics } \\
\text { in childhood }\end{array}$} & Age of polio onset & \\
\hline & $<1$ & $2.5 \%$ \\
\hline & $1-3$ & $85 \%$ \\
\hline & $>3$ & $12.5 \%$ \\
\hline & Weakness in limbs & \\
\hline & Arms or legs & $79.3 \%$ \\
\hline & Both arms and legs & $20.7 \%$ \\
\hline & Trouble breathing in childhood & $25.9 \%$ \\
\hline & Orthopedic surgery in childhood & $71.6 \%$ \\
\hline & Orthotics use in childhood & $61.7 \%$ \\
\hline \multirow{5}{*}{$\begin{array}{l}\text { Poliomyelitis } \\
\text { characteristics } \\
\text { in } 2009\end{array}$} & Scoliosis & $54.4 \%$ \\
\hline & Use of wheelchair & $33 \%$ \\
\hline & Wheelchair bound & $16 \%$ \\
\hline & Use of motorized wheelchair & $13.4 \%$ \\
\hline & Orthotics use in adulthood & $61.0 \%$ \\
\hline
\end{tabular}

such as hypertension, diabetes, and heart diseases. More than $60 \%$ suffered from chronic joint and/or muscular pain. Regarding the onset of poliomyelitis, $85 \%$ were infected with paralytic poliomyelitis between one to three years of age, and almost $20 \%$ presented with paralysis of both upper and lower limbs. More than $70 \%$ underwent orthopedic intervention for limb or spine correction, and more than $60 \%$ needed orthotics devices, such as ankle foot orthoses (AFO) or knee ankle foot orthoses (KAFO) in childhood. In 2009, more than $60 \%$ used AFO or KAFO orthoses, and one third used wheelchairs. 


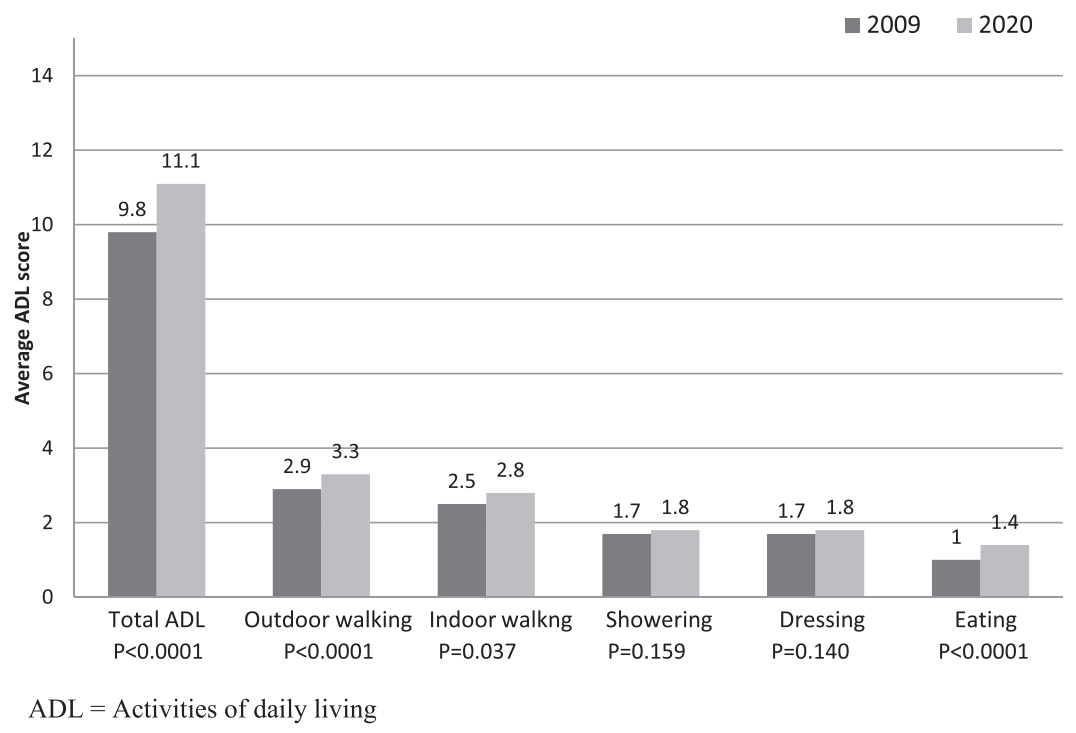

Fig. 1. Comparison of ADL function of 82 poliomyelitis patients followed up for ten years, 2009 and 2020.

\subsection{Comparison of ADL function of 82 polio patients followed up for ten years}

The change in ADL functions of 82 participants during ten years follow-up is presented in Fig. 1. Total ADL worsened significantly, reflecting the worsening of LEoP symptoms. Outdoor walking, indoor walking, and eating deteriorated significantly, whereas bathing and dressing did not change.

\subsection{Association between demographic parameters, comorbidities, poliomyelitis characteristics, and the average change in ADL between 2009 and 2020}

The associations between the various demographic parameters, comorbidities, and poliomyelitis characteristics, and the change in ADL functions are presented in Table 3 . The only significant parameters that affected the deterioration in ADL were ethnicity and the use of a wheelchair in 2009. Reports of the use of orthotics in childhood was also related to greater deterioration in ADL, but it did not reach statistical significance. None of the other parameters correlated with deterioration in ADL.

\subsection{Regression analysis of risk factors for deterioration in $A D L$}

To determine the effect of specific variables on the deterioration in ADL functions, we conducted a regression analysis. As shown in Table 4A, older age and non-Jewish ethnicity were the only demographic parameters that were associated with greater deterioration in ADL functions. All other variables showed no correlation with the deterioration (data not shown). Of the poliomyelitis-related parameters, only the use of orthotics in childhood and use of a wheelchair in 2009 were associated significantly with deterioration in ADL functions in 2020 (Table 4B). None of the other poliomyelitis characteristics correlated with the deterioration. When we add all the parameters with statistical significance above 0.3 into the model, ethnicity, the use of orthotics in childhood and the use of a wheelchair remained significantly associated with deterioration in ADL functions, whereas age was only marginally associated (Fig. 2).

\section{Discussion}

This is the first longitudinal study of poliomyelitis patients with a ten-year follow-up in Israel. We found that patients with LEoP deteriorated significantly in ADL functions, mainly in outdoor and indoor walking ability. The risk factors for deterioration in ADL functions were older age, non-Jewish ethnicity, the use of orthotics in childhood, and the use of a wheelchair in adulthood. By contrast, general comorbidities and other poliomyelitis characteristics did not correlate with ADL deterioration. 
Table 3

Associations between demographic parameters, comorbidities, poliomyelitis characteristics, and the average change in ADL between 2009 and 2020

\begin{tabular}{|c|c|c|c|c|c|}
\hline \multirow[t]{3}{*}{ Characteristics in 2009} & \multicolumn{4}{|c|}{ Average change in total ADL } & \multirow[t]{3}{*}{$p$-value } \\
\hline & \multicolumn{2}{|c|}{ Yes } & \multicolumn{2}{|c|}{ No } & \\
\hline & $\mathrm{N}$ & Mean (SD) & $\mathrm{N}$ & Mean (SD) & \\
\hline \multicolumn{6}{|l|}{ Demographics and vocational } \\
\hline Male & 43 & $1.51(2.55)$ & 39 & $0.97(2.95)$ & 0.3791 \\
\hline Age $\geq 60$ & 35 & $1.31(3.1)$ & 47 & $1.21(2.48)$ & 0.8696 \\
\hline Married & 58 & $1.05(2.45)$ & 24 & $1.75(3.35)$ & 0.3624 \\
\hline Non-Jewish ethnicity & 17 & $2.47(2.58)$ & 65 & $0.94(2.71)$ & 0.0396 \\
\hline Education $>12$ years & 44 & $1.45(2.91)$ & 37 & $1.05(2.59)$ & 0.5183 \\
\hline No physical activity & 49 & $1.55(2.99)$ & 33 & $0.91(2.34)$ & 0.3022 \\
\hline Work in the previous week & 35 & $1.31(2.84)$ & 44 & $1.16(2.78)$ & 0.8232 \\
\hline \multicolumn{6}{|l|}{ Comorbidities } \\
\hline Hypertension & 31 & $1.77(2.94)$ & 49 & $0.88(2.58)$ & 0.1025 \\
\hline Diabetes & 23 & $1.04(2.96)$ & 57 & $1.3(2.67)$ & 0.664 \\
\hline Heart attack & 6 & $2(4)$ & 74 & $1.16(2.64)$ & 0.2394 \\
\hline IHD & 24 & $1.71(3.24)$ & 56 & $1.02(2.5)$ & 0.2172 \\
\hline Lung disease & 9 & $1.56(2.4)$ & 71 & $1.18(2.79)$ & 0.8156 \\
\hline Thyroid disease & 16 & $1.19(2.66)$ & 64 & $1.23(2.78)$ & 0.9121 \\
\hline Pain & 51 & $1.16(2.91)$ & 29 & $1.34(2.47)$ & 0.9132 \\
\hline Obesity & 18 & $1.17(2.28)$ & 62 & $1.24(2.88)$ & 0.8767 \\
\hline Current smoking & 17 & $0.94(3.07)$ & 63 & $1.3(2.67)$ & 0.6507 \\
\hline Past smoking & 30 & $1.63(2.75)$ & 46 & $0.83(2.6)$ & 0.1859 \\
\hline \multicolumn{6}{|l|}{ History of poliomyelitis } \\
\hline Scoliosis & 43 & $0.95(2.78)$ & 36 & $1.56(2.8)$ & 0.3422 \\
\hline Weakness in both arms and legs & 17 & $2.24(2.63)$ & 65 & $1.03(2.74)$ & 0.1083 \\
\hline Trouble breathing & 21 & $0.86(2.26)$ & 58 & $1.40(2.97)$ & 0.4520 \\
\hline Orthopedic surgery in childhood & 58 & $1.16(2.65)$ & 23 & $1.61(3.07)$ & 0.5094 \\
\hline Orthotics use in childhood & 50 & $1.76(2.62)$ & 31 & $0.65(2.80)$ & 0.0733 \\
\hline \multicolumn{6}{|l|}{ Functional characteristics } \\
\hline Usage of a wheelchair & 26 & $2.65(3.45)$ & 53 & $0.53(2.1)$ & 0.0053 \\
\hline Wheelchair bound & 11 & $1.73(1.35)$ & 68 & $1.15(2.95)$ & 0.2343 \\
\hline Motorized wheelchair & 9 & $1.11(0.93)$ & 70 & $1.24(2.95)$ & 0.967 \\
\hline Orthotics use in adulthood & 50 & $1.56(2.61)$ & 32 & $0.84(2.94)$ & 0.2524 \\
\hline
\end{tabular}

Table 4

Regression analysis of risk factors for deterioration in ADL: A. Demographic variables,

B. Poliomyelitis-related variables

\begin{tabular}{|c|c|c|c|}
\hline \multicolumn{4}{|l|}{ A. Demographic variables } \\
\hline Variable & Parameter (SD) & $95 \% \mathrm{CI}$ & $P$-value \\
\hline Gender: Male vs. Female & $0.09(0.66)$ & $(-1.23,1.41)$ & 0.892 \\
\hline Age & $0.11(0.05)$ & $(0.02,0.2)$ & 0.015 \\
\hline Married in 2009 & $-0.65(0.66)$ & $(-1.97,0.67)$ & 0.327 \\
\hline Ethnicity: Jewish vs. Non-Jewish & $-3.14(1.04)$ & $(-5.2,-1.07)$ & 0.003 \\
\hline Education $>12$ years & $0.11(0.62)$ & $(-1.12,1.35)$ & 0.855 \\
\hline Work in the previous week & $0.64(0.65)$ & $(-0.66,1.94)$ & 0.330 \\
\hline Physically active & $0.08(0.63)$ & $(-1.17,1.34)$ & 0.898 \\
\hline \multicolumn{4}{|l|}{ B. Polio-related variables } \\
\hline Variable & Parameter (SD) & $95 \% \mathrm{CI}$ & $P$-value \\
\hline Polio age & $0(0.11)$ & $(-0.22,0.22)$ & 0.988 \\
\hline Weakness: both both arms and legs & $1.18(0.77)$ & $(-0.34,2.71)$ & 0.127 \\
\hline Scoliosis & $1.18(0.65)$ & $(-0.12,2.48)$ & 0.075 \\
\hline Breathing problems & $-0.07(0.71)$ & $(-1.49,1.35)$ & 0.923 \\
\hline Orthopedic surgery in childhood & $1.11(0.76)$ & $(-0.4,2.62)$ & 0.147 \\
\hline Orthotics use in childhood & $-1.44(0.68)$ & $(-2.79,-0.09)$ & 0.037 \\
\hline Orthotics use in adulthood & $-0.81(0.63)$ & $(-2.07,0.45)$ & 0.204 \\
\hline Use of wheelchair & $1.93(0.67)$ & $(0.59,3.28)$ & 0.006 \\
\hline
\end{tabular}

$\mathrm{ADL}=$ Activities of daily living. 


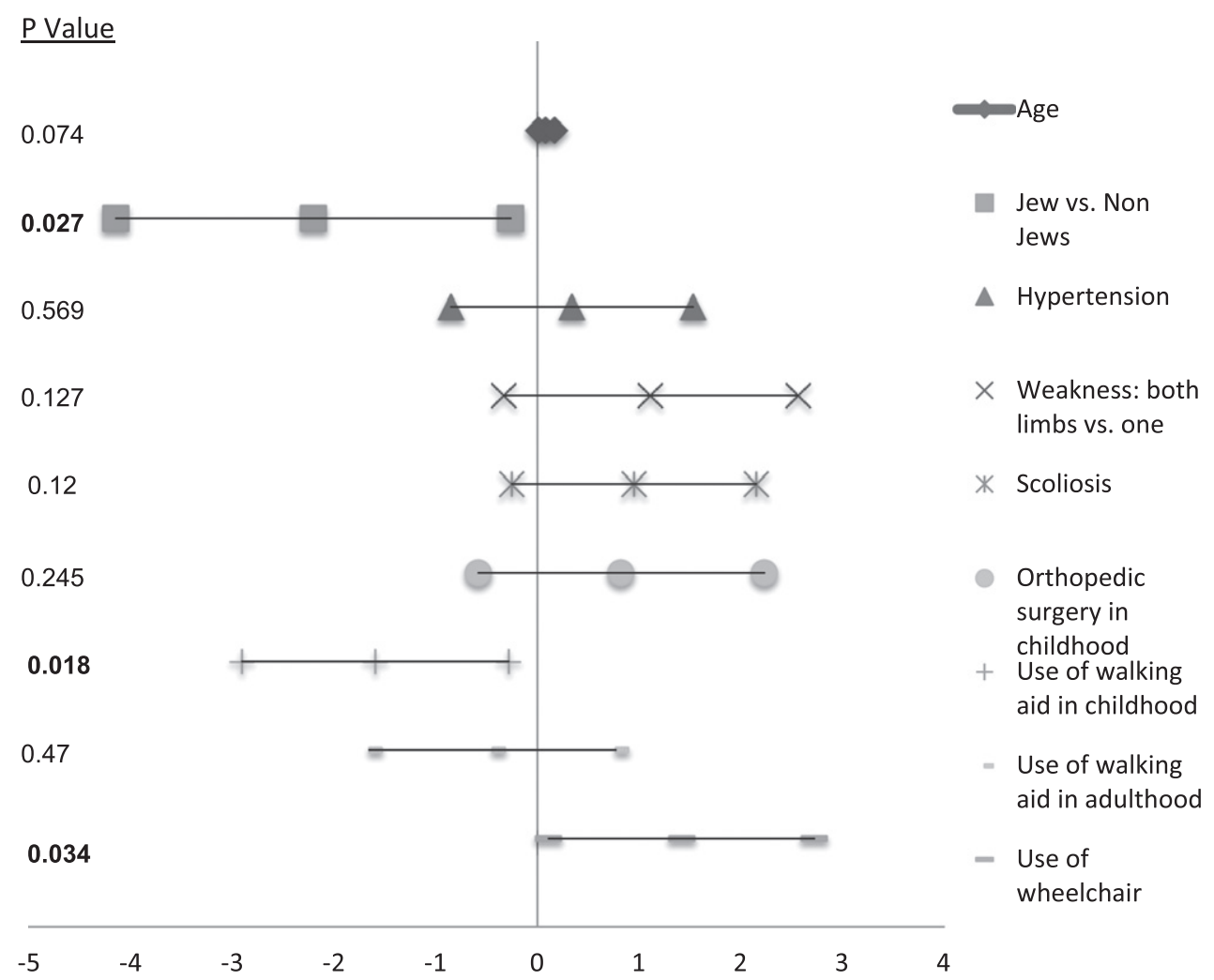

Fig. 2. Regression analysis of risk factors for deterioration in ADL.

In our previous study, we found that people with LEoP were significantly less independent in their ambulatory capacity indoors and outdoors, and in their ADL functioning than was the general population (Schwartz et al., 2018). Our present study shows that the functional level of these patients ten years later deteriorated significantly, and the difference between them and the general population became even more significant. This finding may reflect the early aging process of people with LEoP, similar to that of other individuals disabled at young age because of congenital or acquired etiologies. The phenomenon of accelerated aging of people with disabilities is the result of the wear-and-tear phenomenon associated with ongoing psychological, biological, and social stressors caused by the life-long struggle to maintain homeostasis (Ohry \& Keren, 2008). Living with LEoP places poliomyelitis survivors at potentially increased risk of age-related chronic diseases and health conditions, such as diabetes, high blood pressure, heart disease, emphysema or COPD (chronic obstructive pulmonary disease), osteoporosis, and obesity (Kang \& Lin, 2011; Schwartz et al., 2018). They also face the effects of developing polio-related symptoms, including muscle weakness, fatigue, and muscle or joint pain owing to the high prevalence of post-polio syndrome ( $\mathrm{Li}$ Hi Shing et al., 2019). The significance of both the post-polio syndrome and of early onset age-related chronic diseases lies in their potential to accelerate the aging process and produce secondary disabilities, as demonstrated in our study (McNalley \& Yorkston \& Jensen \& Truitt \& Schomer, 2015). Medical and rehabilitation intervention, together with proper health education may delay these premature aging processes in this population.

\subsection{Effect of ethnicity on poliomyelitis}

Our study also showed that individuals of ethnic minority with LEoP displayed greater deterioration in functional level than did the ethnic majority 
population. Similar finding was observed in a study from Sweden, in which minority patients with LEoP had more severe forms of the diseases than did native Swedish patients (Werhagen \& Borg, 2016). Moreover, a similar difference in level of functional dependence was found between poliomyelitis patients from countries with marked difference in socioeconomic status: Norwegians and Estonians (Rekand et al., 2003). A possible explanation of such differences between ethnic populations of people with LEoP lies in differences in cultural and socioeconomic status.

\subsection{Effect of poliomyelitis history and characteristics on ADL deterioration}

The study found that only the use of orthotics in childhood was associated with the functional deterioration of people with LEoP. All other parameters related to the history of poliomyelitis, including age at initial infection, extent of limb involvement, presence of scoliosis or breathing problems, and orthopedic surgery in childhood did not correlate with the progression of LEoP. Similarly to our previous study, we found no correlation between age at polio onset and the severity of polio sequelae (Schwartz et al., 2014). Other studies reported a similar absence of association (Nollet et al., 2002; Trojan \& Cashman \& Shapiro \& Tansey \& Esdaile, 1994), except for one study that found an association between post-polio syndrome and early age of polio onset (Ragonese et al., 2005). We suggest that age at the onset of paralytic poliomyelitis has a minor effect of functional deterioration in people with LEoP. By contrast, according to our results, severity of the primary neurological involvement, as reflected in need for orthotics in childhood and the use of a wheelchair in adulthood, attesting to severe neurological involvement, had a significant effect on functional deterioration. The use of orthotics in adulthood, however, was not correlated with higher deterioration in ADL functions because orthotic devices are usually prescribed to restore function and prevent falls. Thus, our findings suggest that the use of orthotics in childhood is a marker of disease severity and has a long-term effect on the functional capacity of individuals with LEoP, whereas orthotics used in adulthood may have some protective effect against functional deterioration (Genêt et al., 2010).

Scoliosis was found in $54.4 \%$ of our patients, compared to $65 \%$ in a cohort of 85 patients with LEoP in Norway (Farbu \& Rekand \& Gilhus, 2003). Respiratory involvement was reported in $25.9 \%$ of the participants in our study, as compared to only $7 \%$ in the Norwegian cohort. Yet, neither of these symptoms was associated with deterioration in ADL functions, suggesting that their severity was not high enough to cause functional deterioration.

\subsection{Comparison with other longitudinal studies of people with LEoP}

Several studies have examined the progression of the disability of people with LEoP and examined the influence of diverse factors on the rate of progression. Three earlier follow-up studies of 15 years (50 subjects), 4 years (106 subjects), and 1 week to 3 years (96 subjects) reported minor-to-modest changes in walking tests, perceived physical mobility, upper limb functioning, and muscle strength (Klein et al., 2008; Sorenson \& Daube \& Windebank, 2005; Willen \& Thoren-Jonsson \& Grimby \& Stibrant Sunnerhagen, 2007). Other more recent studies found similar deterioration over 5 to ten years (Bickerstaffe \& Beelen \& Nollet, 2015; Flansbjer \& Brogardh \& Horstmann \& Lexell, 2015; Stolwijk-Swüste \& Tersteeg \& Beelen \& Lankhorst \& Nollet, 2010). Stolwijk-Swüste et al. described the functional course over 5 years of 168 patients aged 45 to 85 years with LEoP and found that despite a reduction in muscle strength, disability increased little during this period (Stolwijk-Swüste \& Tersteeg \& Beelen \& Lankhorst $\&$ Nollet, 2010). In their study, similar to our finding, increased age had a negative effect on disability, attesting to the phenomenon of accelerated aging in people with LEoP. In this study, as opposed to our finding, comorbidity affected the course of functional independence, but only the cumulative effect of comorbidities, as measured by the cumulative illness rating scale (CIRS), was associated with greater deterioration in functioning, not the specific comorbidities examined in our study. The rate of functional deterioration was examined using the progression in the Functional Independence Measure (FIM) score, which was $1.8 \%$ over 5 years and occurred in the motor items of the instrument. In our study, the rate of progression over 10 years was markedly higher, $13 \%$, and it was manifest mainly in the daily mobility functioning of indoor and outdoor walking, not in personal care. This difference can be explained by the fact that we used a self-reporting scale for ADL evaluation, and they used the FIM index, which is based on caregiver observation. Another possible explanation is that according to a comparison between functional scores of the two cohorts, ours 
was more disabled, whereas the Stolwijk-Swüste's cohort scored in the upper range of FIM, reflecting minimal disability (Stolwijk-Swüste \& Tersteeg \& Beelen \& Lankhorst \& Nollet, 2010). Another factor that contributed to the functional deterioration in the Stolwijk-Swüste's study was the severity of leg paresis, this may be equivalent to our finding that people with LEoP who used wheelchairs, deteriorated significantly more than those who did not use wheelchairs.

\subsection{Limitations of the study}

Our study has several limitations. The number of patients who participated in both the original and the present study was relatively small, reflecting the attrition over the ten-year period between the two surveys and the difficulties in reaching all patients. Furthermore, poliomyelitis patients who attend our post-polio clinic present with more severe symptoms than do Israeli poliomyelitis patients in general, therefore a selection bias may reduce the external validity of the findings. Our survey was conducted by phone and used self-report scales, therefore it shares the limitation of other studies using a similar method. Yet, the ten-year within-subject design of the study enabled us to obtain important and unique information about the state of individuals with LEoP.

\section{Conclusions}

The results of the present study indicate that aging has a significant effect on the independence and quality of life of people with LEoP. Other risk factors for deterioration in functional independence were ethnicity, use of a wheelchair, and use of an orthotic device in childhood. These findings emphasize the need for specific rehabilitation programs for to prevent further deterioration in this population.

\section{Conflict of interest}

None of the authors have any conflict of interest to declare.

\section{Funding}

This work was supported by a grant from the Israeli National Insurance Institute.

\section{References}

Adler, E., Eliakim, C., \& Magora, A. (1955). Poliomyelitis in Jerusalem in 1953. Acta Medica Orientalia, 14(6), 147-163.

Ahstrom, G., \& Karlsson, U. (2000). Disability and quality of life in individuals with postpolio syndrome. Disabil Rehabil, 22, 416-422.

Bickerstaffe, A., Beelen, A., \& Nollet, F. (2015). Change in physical mobility over 10 years in post-polio syndrome. $\mathrm{Neu}$ romuscular Disorders, 25(3), 225-230.

Dalakas, M. C., Elder, G., Hallett, M., Ravits, J., Baker, M., Papadopoulos, N.,... \& Sever, J. (1986). A long-term follow-up study of patients with post-poliomyelitis neuromuscular symptoms. New England Journal of Medicine, 314(15), 959-963.

Farbu, E., Rekand, T., \& Gilhus, N. E. (2003). Post-polio syndrome and total health status in a prospective hospital study. European Journal of Neurology, 10(4), 407-413.

Flansbjer, U. B., Brogårdh, C., Horstmann, V., \& Lexell, J. (2015). Men with late effects of polio decline more than women in lower limb muscle strength: a 4-year longitudinal study. $P M \& R, 7(11), 1127-1136$.

Genêt, F., Schnitzler, A., Mathieu, S., Autret, K., Théfenne, L., Dizien, O., \& Maldjian, A. (2010). Orthotic devices and gait in polio patients. Annals of Physical and Rehabilitation Medicine, 53(1), 51-59.

Gonzalez, H., Olsson, T., \& Borg, K. (2010). Management of postpolio syndrome. The Lancet Neurology, 9(6), 634-642.

Halstead, L. S. (2011). A brief history of postpolio syndrome in the United States. Archives of Physical Medicine and Rehabilitation, 92(8), 1344-1349.

Jacob, T., \& Shapira, A. (2010). Quality of life and health conditions reported from two post-polio clinics in Israel. Journal of Rehabilitation Medicine, 42(4), 377-379.

Kang, J. H., \& Lin, H. C. (2011). Comorbidity profile of poliomyelitis survivors in a Chinese population: a populationbased study. Journal of Neurology, 258(6), 1026-1033.

Klein, M. G., Braitman, L. E., Costello, R., Keenan, M. A., \& Esquenazi, A. (2008). Actual and perceived activity levels in polio survivors and older controls: a longitudinal study. Archives of Physical Medicine and Rehabilitation, 89(2), 297-303.

Li Hi Shing, S., Chipika, R. H., Finegan, E., Murray, D., Hardiman, O., \& Bede, P. (2019). Post-polio syndrome: more than just a lower motor neuron disease. Frontiers in Neurology, 10, 773.

Lo, J. K., \& Robinson, L. R. (2018). Postpolio syndrome and the late effects of poliomyelitis. Part 1. pathogenesis, biomechanical considerations, diagnosis, and investigations. Muscle \& Nerve, 58(6), 751-759.

McNalley, T. E., Yorkston, K. M., Jensen, M. P., Truitt, A. R., Schomer, K. G., Baylor, C., \& Molton, I. R. (2015). A review of secondary health conditions in post-polio syndrome: Prevalence and effects of aging. American Journal of Physical Medicine \& Rehabilitation/Association of Academic Physiatrists, 94(2), 139.

Nielsen, N. M., Rostgaard, K., Askgaard, D., Skinhøj, P., \& Aaby, P. (2004). Life-long morbidity among Danes with poliomyelitis. Archives of Physical Medicine and Rehabilitation, 85(3), 385-391.

Nollet, F., Ivanyi, B., Beelen, A., De Haan, R. J., Lankhorst, G. J., \& De Visser, M. (2002). Perceived health in a population based sample of victims of the 1956 polio epidemic in the 
Netherlands. Journal of Neurology, Neurosurgery \& Psychiatry, 73(6), 695-700.

Ohry, A., \& Keren, O. (2008). The premature-aging process and new functional losses among people with chronic disabilities. Critical Reviews $^{\mathrm{TM}}$ in Physical and Rehabilitation Medicine, 20(1).

Pfeiffer, E. (1975). A short portable mental status questionnaire for the assessment of organic brain deficit in elderly patients. Journal of the American Geriatrics Society, 23(10), 433-441.

Ragonese, P., Fierro, B., Salemi, G., Randisi, G., Buffa, D., D'Amelio, M.,... \& Savettieri, G. (2005). Prevalence and risk factors of post-polio syndrome in a cohort of polio survivors. Journal of the Neurological Sciences, 236(1-2), 31-35.

Rekand, T., Kõrv, J., Farbu, E., Roose, M., Gilhus, N. E., Langeland, N., \& Aarli, J. A. (2003). Long term outcome after poliomyelitis in different health and social conditions. Journal of Epidemiology \& Community Health, 57(5), 368-372.

Schwartz, I., Gartsman, I., Adler, B., Friedlander, Y., Manor, O., Levine, H., \& Meiner, Z. (2014). The association between post-polio symptoms as measured by the Index of Post-Polio Sequelae and self-reported functional status. Journal of the Neurological Sciences, 345(1-2), 87-91.

Schwartz, I., Gartsman, I., Adler, B., Friedlander, Y., Manor, O., Levine, H., \& Meiner, Z. (2018). High Prevalence of Cardiovascular Comorbidity and Chronic Pain in Polio Survivors as Compared to the General Population. Physical Medicine and Rehabilitation - International, 5(1), 1138-1145.
Slater, P. E., \& HANDSHER, R. (1989). Polio in Israel. Nature, $338(6211), 110-110$.

Sorenson, E. J., Daube, J. R., \& Windebank, A. J. (2005). A 15year follow-up of neuromuscular function in patients with prior poliomyelitis. Neurology, 64(6), 1070-1072.

Stolwijk-Swüste, J. M., Tersteeg, I., Beelen, A., Lankhorst, G. J., Nollet, F., \& CARPA Study Group. (2010). The impact of age and comorbidity on the progression of disability in late-onset sequelae of poliomyelitis. Archives of Physical Medicine and Rehabilitation, 91(4), 523-528.

Swartz, T. A. (2008). The epidemiology of polio in Israel. Dyonon Pub. Ltd.

Trojan, D. A., \& Cashman, N. R. (2005). Post-poliomyelitis syndrome. Muscle \& Nerve: Official Journal of the American Association of Electrodiagnostic Medicine, 31(1), 6-19.

Trojan, D. A., Cashman, N. R., Shapiro, S., Tansey, C. M., \& Esdaile, J. M. (1994). Predictive factors for post-poliomyelitis syndrome. Archives of Physical Medicine and Rehabilitation, 75(7), 770-777.

Werhagen, L., \& Borg, K. (2016). Survey of young patients with polio and a foreign background at a Swedish post-polio outpatient clinic. Neurological Sciences, 37(10), 1597-1601.

Willen, C., Thoren-Jönsson, A. L., Grimby, G., \& Sunnerhagen, K. S. (2007). Disability in a 4-year follow-up study of people with post-polio syndrome. Journal of Rehabilitation Medicine, 39(2), 175-180. 